\title{
Sensitized mutagenesis screen in Factor V Leiden mice identifies thrombosis suppressor loci
}

\author{
Randal J. Westrick ${ }^{a, b, c, 1,2}$, Kärt Tomberg ${ }^{c, d, 1}$, Amy E. Siebert ${ }^{a, 1}$, Guojing Zhuc, Mary E. Winn ${ }^{\mathrm{e}}$, Sarah L. Dobiesc, \\ Sara L. Manning c, Marisa A. Brake ${ }^{a}$, Audrey C. Cleurenc', Linzi M. Hobbs ${ }^{a}$, Lena M. Mishacka, Alexander J. Johnston ${ }^{a}$,

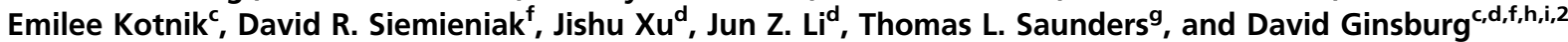

a Department of Biological Sciences, Oakland University, Rochester, MI 48309; ${ }^{\circ}$ Center for Data Science and Big Data Analysis, Oakland University, Rochester,

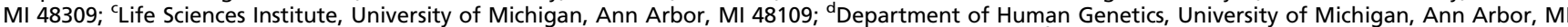
48109; 'Bioinformatics and Biostatistics Core, Van Andel Research Institute, Grand Rapids, MI 49503; ${ }^{f}$ Howard Hughes Medical Institute, University of

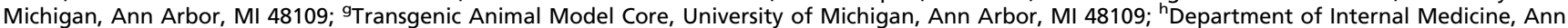
Arbor, MI 48109; and 'Department of Pediatrics, University of Michigan, Ann Arbor, MI 48109

Contributed by David Ginsburg, July 24, 2017 (sent for review April 7, 2017; reviewed by Monica J. Justice and Joost Meijers)

Factor $\mathrm{V}$ Leiden $\left(F 5^{L}\right)$ is a common genetic risk factor for venous thromboembolism in humans. We conducted a sensitized $\mathrm{N}$-ethyl- $\mathrm{N}$ nitrosourea (ENU) mutagenesis screen for dominant thrombosuppressor genes based on perinatal lethal thrombosis in mice homozygous for $F 5^{L}\left(F 5^{L /}\right)$ and haploinsufficient for tissue factor pathway inhibitor $\left(\mathrm{Tfpi}^{+/-}\right)$. F8 deficiency enhanced the survival of $\mathrm{F5}^{L / L} \mathrm{Tfpi}^{+/-}$mice, demonstrating that $\mathrm{F5}^{L / L} \mathrm{Tfpi}^{+/-}$lethality is genetically suppressible. ENU-mutagenized $F 5^{L / L}$ males and $\mathrm{FS}^{L+} \mathrm{Tfpi}^{+/-}$females were crossed to generate 6,729 progeny, with $98 \mathrm{F5}^{L / L} \mathrm{Tfpi}^{+/-}$offspring surviving until weaning. Sixteen lines, referred to as "modifier of Factor 5 Leiden (MF5L1-16)," exhibited transmission of a putative thrombosuppressor to subsequent generations. Linkage analysis in MF5L6 identified a chromosome 3 locus containing the tissue factor gene (F3). Although no ENU-induced $F 3$ mutation was identified, haploinsufficiency for $\mathrm{F3}\left(\mathrm{F3}^{+/-}\right)$suppressed $\mathrm{F5}^{L /} \mathrm{Tfpi}^{+/-}$lethality. Whole-exome sequencing in MF5L12 identified an Actr2 gene point mutation (p.R258G) as the sole candidate. Inheritance of this variant is associated with suppression of $F 5^{L / L} \mathrm{Tfpi}^{+/-}$lethality $\left(P=1.7 \times 10^{-6}\right)$, suggesting that Actr2 $^{\text {p.R258G }}$ is thrombosuppressive. CRISPR/Cas9 experiments to generate an independent Actr2 knockin/knockout demonstrated that Actr2 haploinsufficiency is lethal, supporting a hypomorphic or gain-of-function mechanism of action for Actr2 ${ }^{\mathrm{p} . \mathrm{R} 258 \mathrm{G}}$. Our findings identify $F 8$ and the $T f$ pi/F3 axis as key regulators in determining thrombosis balance in the setting of $F 5^{L}$ and also suggest a role for Actr2 in this process.

venous thromboembolism | Factor V Leiden | ENU mutagenesis | tissue factor pathway inhibitor | genetic screen

V enous thromboembolism (VTE) is a common disease that affects 1-3 per 1,000 individuals each year (1). VTE susceptibility exhibits a complex etiology involving contributions of both genes and environment. Genetic risk factors explain $\approx 60 \%$ of the overall risk for VTE (2). Recent large-scale genome-wide association studies (GWAS) confirmed $A B O, F 2 F 5, F 11, F G G$, and PROCR as thrombosis susceptibility genes, with only two additional novel loci, TSPAN15 and SLC44A2, identified (3-6), leaving the major component of VTE genetic risk still unexplained.

The Factor V Leiden variant $\left(F 5^{L}\right)$ is a common inherited risk factor for VTE with an average allele frequency of $3.5 \%$ in the European population (7-9). $F 5^{L}$ is estimated to account for up to $25 \%$ of the genetically attributable thrombosis risk in this population (7). However, penetrance is incomplete, with only $\sim 10 \%$ of $F 5^{L}$ heterozygotes developing thrombosis in their lifetimes. The severity of thrombosis also varies widely among affected individuals (10), limiting the clinical utility of $F 5^{L}$ genotyping in the management of VTE (11).

The incomplete penetrance and variable expressivity of thrombosis among $F 5^{L}$ patients can at least partially be explained by genetic interactions between $F 5^{L}$ and other known thrombotic risk factors such as hemizygosity for antithrombin III or proteins $\mathrm{C}$ or $\mathrm{S}$, as well as the common prothrombin 20210 polymorphism (10, 12,
13). However, $<2 \%$ of $F 5^{L}$ heterozygotes would be expected to coinherit a mutation at one or more of these loci, suggesting that a large number of additional genetic risk factors for VTE and/or modifiers of $F 5^{L}$ remain to be identified $(3,10)$.

Mice carrying the orthologous $F 5^{L}$ mutation exhibit a mild to moderate prothrombotic phenotype closely mimicking the human disorder (14). We previously reported a synthetic lethal interaction between $F 5^{L}$ homozygosity $\left(F 5^{L / L}\right)$ and hemizygosity for tissue factor pathway inhibitor $\left(T f p i^{+-}\right)(15)$. Nearly all mice with this lethal genotype combination $\left(F 5^{L / L} T f p i^{+/-}\right)$succumb to widespread, systemic thrombosis in the immediate perinatal period (15).

$N$-ethyl- $N$-nitrosourea (ENU) mutagenesis in mice has been used effectively to identify novel genes involved in a number of biological processes $(16,17)$. ENU-induced germline mutations transmitted from a mutagenized male mouse (G0) occur at $\sim 1.5$ mutations per megabase, at least 50-fold higher than the endogenous background mutation rate (18). Several previous reports have successfully applied an existing phenotype as a sensitizer to identify modifier genes. A dominant suppressor screen in Mecp2-deficient mice (Rett syndrome) identified a mutation in squalene epoxidase (Sqle) as a heritable suppressor, resulting in prolonged survival and amelioration of neurologic manifestations (19). Other successful sensitized screens include analysis of mouse mutants predisposed to diabetic nephropathy (20), a screen in Sox10 haploinsufficient mice identifying the Gli3 gene as a modifier of neurocristopathy

\section{Significance}

Venous thromboembolism (VTE) is a common disease characterized by the formation of inappropriate blood clots. Inheritance of specific genetic variants, such as the Factor $\mathbf{V}$ Leiden polymorphism, increases VTE susceptibility. However, only $\sim 10 \%$ of people inheriting Factor V Leiden develop VTE, suggesting the involvement of other genes that are currently unknown. By inducing random genetic mutations into mice with a genetic predisposition to VTE, we identified two genomic regions that reduce VTE susceptibility. The first includes the gene for blood coagulation, Factor 3, and its role was confirmed by analyzing mice with an independent mutation in this gene. The second contains a mutation in the Actr2 gene. These findings identify critical genes for the regulation of blood-clotting risk.

Author contributions: R.J.W., K.T., A.E.S., and D.G. designed research; R.J.W., K.T., A.E.S., G.Z., M.E.W., S.L.D., S.L.M., M.A.B., A.C.C., L.M.H., L.M.M., A.J.J., E.K., D.R.S., and T.L.S. performed research; R.J.W., K.T., A.E.S., G.Z., M.E.W., S.L.D., A.C.C., E.K., D.R.S., J.X., J.Z.L., T.L.S., and D.G. analyzed data; and R.J.W., K.T., A.E.S., and D.G. wrote the paper. Reviewers: M.J.J., The Hospital for Sick Children; and J.M., Sanquin Research.

The authors declare no conflict of interest.

${ }^{1}$ R.J.W., K.T., and A.E.S. contributed equally to this work.

${ }^{2}$ To whom correspondence may be addressed. Email: ginsburg@umich.edu or rjwestrick@ oakland.edu.

This article contains supporting information online at www.pnas.org/lookup/suppl/doi:10. 1073/pnas.1705762114/-/DCSupplemental. 
(21), and identification of a mutation in the $c-M y b$ gene as a dominant modifier for platelet count in $\mathrm{Mpl}$-deficient mice (congenital thrombocytopenia) (22). We now report the results of a dominant, sensitized ENU mutagenesis screen for suppressors of $F 5^{L / L} T f p i^{+/-}$-dependent lethal thrombosis.

\section{Results and Discussion}

F8 Deficiency Suppresses $\boldsymbol{F 5}^{\mathbf{L} / \mathbf{L}} \mathbf{T f} \boldsymbol{i}^{+/-}$Lethality. X-linked hemophilia A results in a moderate-to-severe bleeding disorder in humans and is caused by mutations in the $F 8$ gene. To test whether the $F 5^{L / L}$ $T f p i^{+/-}$lethal thrombotic phenotype is suppressible by hemophilia A in mice, triple-heterozygous $F 5^{L /+} T f p i^{+/-} F 8^{+/-}$female mice were generated and crossed to $F 5^{L / L}$ male mice (Fig. $1 A$ ). One quarter of conceptuses are expected to carry the $F 5^{L / L} T f p i^{+/-}$genotype, with half of the total expected male conceptuses completely $F 8$ deficient $\left(F 8^{-}\right)$. Thus, 1/16th of the overall offspring from this mating are expected to be $F 5^{L / L} T f p i^{+/-} F 8^{-}$males. Similarly, 1/16th of the progeny should be $F 5^{L / L} \mathrm{Tfpi}^{+/-} F 8^{+/-}$females. A total of 163 progeny from this cross were genotyped at weaning, resulting in eight $F 5^{L / L} T f p i^{+/-} F 8^{-}$male mice observed (and $0 F 5^{L / L} \mathrm{Tfpi}^{+/-} \mathrm{F8^{+ }}$ male mice, $P=0.02$ ) and two $F 5^{L / L} T f p i^{+/-} F 8^{+/-}$female mice (and one $F 5^{L / L} \mathrm{Tfpi}^{+/-} F 8^{+/+}$female mouse, $P=0.9$ ). These results demonstrate that $F 5^{L / L} \mathrm{Tfpi}^{+/-}$thrombosis is genetically suppressible by $F 8$ deficiency with nearly complete penetrance in $\mathrm{F}^{-}$male mice and are consistent with human studies demonstrating $F 8$ level as an important VTE risk factor (23).

The $\mathrm{FF}^{\mathrm{L} / \mathbf{L}} \mathrm{Tfpi}^{+/-}$Phenotype Is Suppressed by Dominant ENU-Induced Mutations. A sensitized, genome-wide ENU mutagenesis screen for dominant thrombosis suppressor genes was implemented as
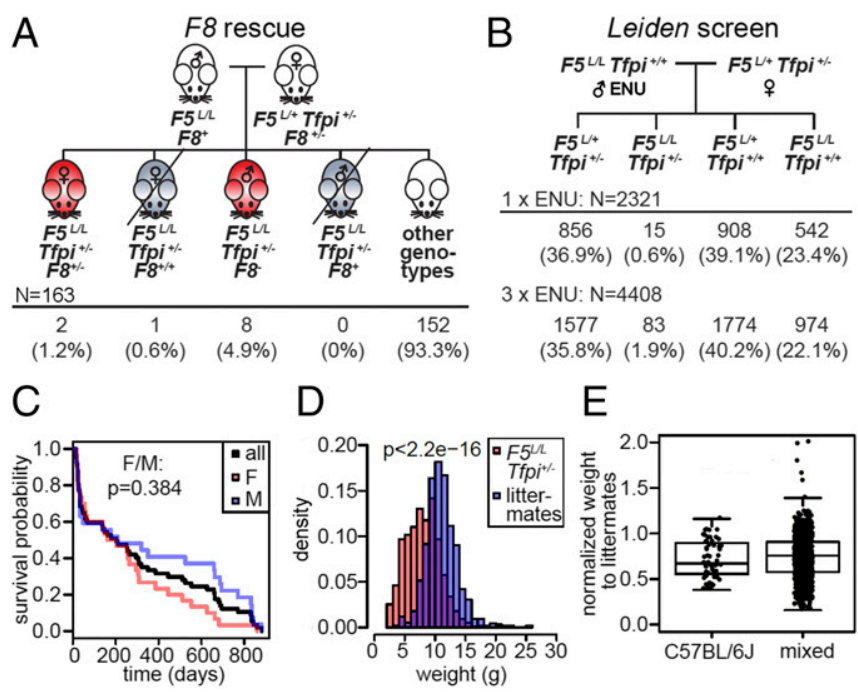

$\mathrm{E}$

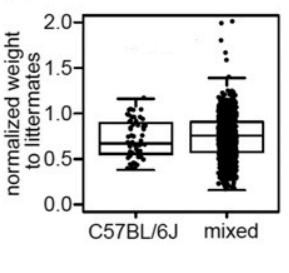

Fig. 1. F8-deficient thrombosuppression and design of the Leiden ENU mutagenesis screen. $(A)$ The mating scheme and observed distributions of the $F 5^{L /+}$ $\mathrm{Tfpi}^{+/-} \mathrm{F8}$ deficiency rescue experiments. $\mathrm{FS}^{-}$results in suppression of the $F 5^{L / L}$ $\mathrm{Tfpi}^{+/-}$phenotype. (B) The mating scheme and observed distribution of the Leiden screen. $\mathrm{FS}^{L / L} \mathrm{Tfpi}^{+/+}$male mice were mutagenized with either $1 \times 150 \mathrm{mg} / \mathrm{kg}$ or $3 \times$ $90 \mathrm{mg} / \mathrm{kg}$ ENU and were bred with nonmutagenized $\mathrm{FS}^{\mathrm{L/}+} \mathrm{Tfpi}^{+/-}$females. Fifteen and eighty-three $\mathrm{F5}^{\mathrm{L} / \mathrm{L}} \mathrm{Tfpi}^{+/-}$progeny, respectively were observed in each of the dosing regimens, with more than twice the rate of $\mathrm{FS}^{\mathrm{L} / L} \mathrm{Tfpi}^{+/-}$survivors in the progeny of the $3 \times 90 \mathrm{mg} / \mathrm{kg}$-treated mice. $(C)$ There was no significant difference in survival between male and female $F 5^{L / L} \mathrm{Tfpi}^{+/-}$putative suppressor mice $(P=$ 0.384 ). Normal weaning and breeding ages are $20 \mathrm{~d}$ and $42 \mathrm{~d}$, respectively. (D) $F 5^{L / L} \mathrm{Tfpi}^{+/-}$putative suppressor mice were significantly smaller than their nonF5 $5^{L / L} \mathrm{Tfpi}^{+/-}$littermates. (E) $\mathrm{FS}^{\mathrm{L} / L} \mathrm{Tfpi}^{+/-}$putative suppressors were smaller than their littermates of other genotypes $\left(P<8.8 \times 10^{-12}\right.$ for $\mathrm{B} 6$ and $P=2.2 \times 10^{-}$ ${ }^{16}$ for mixed B6-129S1) regardless of whether they were on the pure B6 or mixed B6-129S1 genetic backgrounds ( $P=0.327$ between $\mathrm{B} 6$ and mixed backgrounds). depicted in Fig. $1 B$. ENU-mutagenized G0 $F 5^{L / L}$ males were crossed to $F 5^{L /+} T f p i^{+-}$females to generate G1 mice, which were screened by genotyping at weaning for $F 5^{L}$ and $T f p i^{+/}$. Previously described visible dominant mutant phenotypes (24), including belly spotting and skeletal abnormalities, were observed in $\approx 5.9 \%$ of G1 offspring, similar to the $\sim 4.2 \%$ rate of observable mutants in previous studies (24). This is consistent with the $\sim 20-30$ functionally significant mutations per G1 mouse expected with this ENU mutagenesis protocol (25). Although $25 \%$ of G1 embryos from this cross are expected to carry the synthetic lethal $F 5^{L / L} T f p i^{+/-}$genotype, most are lost at birth. Given a total of $6,631 \mathrm{G} 1 \mathrm{~s}$ for the other three genotypes observed at weaning ( $\sim 1 / 3$ for each genotype), a similar number of $F 5^{L / L} \mathrm{Tfpi}^{+/-} \mathrm{G} 1$ conceptuses, 2,210 (6,631 $\div 3$ ), would have been expected. The 98 live $F 5^{L / L} \mathrm{Tfpi}^{+/-}$mice (45 females, 53 males) thus represented $4.4 \%$ of the number expected with this genotype. Survival data were collected for 57 of the $F 5^{L / L} \mathrm{Tfpi}^{+/-}$ G1 mice, 34 of which lived past $70 \mathrm{~d}$ of age; precise dates of death were not available for the remaining 41 mice. No significant sexspecific differences in survival were observed (Fig. 1C).

Heritability for each of the $44 \mathrm{G} 1$ putative suppressor mutants who lived to breeding age was evaluated by a progeny test backcross to $\mathrm{C} 57 \mathrm{BL} / 6 \mathrm{~J}$ (B6) $F 5^{L / L}$ mice. The observation of one or more $F 5^{L / L} \mathrm{Tfpi}^{+/-}$offspring surviving to weaning increased the likelihood that a particular modifier of Factor 5 Leiden $(M F 5 L)$ line carries a transmissible suppressor mutation. Of the original 98 surviving $F 5^{L / L} T_{f p i}{ }^{+/-}$G1 mice, 75 produced no offspring surviving to weaning, either due to infertility or the abovementioned early lethality, with $>50 \%$ of these mice (37 of 75) exhibiting a grossly runted appearance. Approximately half of the $F 5^{L / L} \mathrm{Tfpi}^{+--} \mathrm{G} 1$ mice that attained breeding age (23/44) produced one or more G2 progeny surviving to weaning; seven (two males and five females) produced no $F 5^{L / L} \mathrm{Tfpi}^{+/-} \mathrm{G} 2 \mathrm{~s}$, including four $\mathrm{G} 1 \mathrm{~s}$ with eight or more offspring of other genotypes. Sixteen $F 5^{L / L}$ $\mathrm{Tfpi}^{+/-} \mathrm{G} 1$ mice produced one or more $F 5^{L / L} \mathrm{Tfpi}^{+/-}$progeny when bred to B6 F5 $5^{L / L}$ mice (Materials and Methods). These 16 potential thrombosuppressor mouse lines are designated MF5L1-16. The number of total progeny, genotypic distribution, and penetrance of the $F 5^{L / L} \mathrm{Tfpi}^{+/-}$mice in each line are listed in Table 1. Within these suppressor lines, mice with the $F 5^{L / L} \mathrm{Tfpi}^{+/-}$genotype were $\sim 30 \%$ smaller than their $F 5^{L / L}$ littermates at the time of weaning $\left(P<2.2 \times 10^{-16}\right)($ Fig. $1 D)$, and this difference was maintained after outcrossing to the 129S1 strain (Fig. 1E).

Previous reports based on gene function in the specific-locus test estimate an ENU-induced mutation rate of 1/700 loss-of-function mutations per locus for the ENU dosing regimen used here (26). This mutation rate predicts that our screen of 6,729 G1 progeny $\left(2,210 F 5^{L / L} T f p i^{+/-}\right.$expected) should have produced approximately three mutations per gene averaged over the entire genome, with $54 \%$ of these mutations expected to be null (16), resulting in $1.5 \times$ genome coverage for loss-of-function mutations.

The MF5L6 Suppressor Mutation Maps to a Chromosome 3 Interval Containing F3. To map putative ENU-induced suppressor mutations, surviving $F 5^{L / L} \mathrm{Tfpi}^{+/-}$mice were intercrossed with $F 5^{L / L}$ mice that had been backcrossed onto the 129S1/SvIMJ strain (129S1). Crosses between $F 5^{L / L}$ and $F 5^{L /+} T f p i^{+-}$mice (both $F 5^{L}$ and Tfpi $^{-}$backcrossed $>12$ generations onto 129S1) confirmed the lethality of the $F 5^{L / L} \mathrm{Tfpi}^{+-}$genotype on the 129S1 background (Table S1). The four lines containing the largest number of genetically informative B6-129S1 mixed background $F 5^{L / L} T f p i^{+/-}$ offspring (MF5L1, 6, 9, and 16) were used for gene mapping. Although the MF5L1, MF5L9, and MF5L16 lines were successfully expanded to pedigrees containing 27,84 , and $14 F 5^{L / L} T f p i^{+/-}$ informative offspring, respectively, genotyping for a total of $\sim 800$ markers in each cross failed to identify any loci with a logarithm of the odds (LOD) score greater than or equal to 3.3 (maximum LODs for MF5L1 = 1.15, MF5L9 $=2.5$, and MF5L16 = 1.61). Although we cannot exclude cosegregation of 
Table 1. Progeny genotypes and penetrance of putative $M F 5 L$ thrombosuppressor genes

\begin{tabular}{|c|c|c|c|c|c|c|}
\hline ENU line & Sex of G1 & $\begin{array}{l}\text { Total no. of } \\
\text { progeny }\end{array}$ & $\begin{array}{c}\text { Total no. of } \\
\text { F5 }^{\mathrm{L} / \mathrm{L}} \mathrm{Tfpi}^{+/-} \text {mice }\end{array}$ & $\begin{array}{c}\text { No. of } F 5^{\mathrm{L} / \mathrm{L}} \\
T f p i^{+/+} \text {littermates }\end{array}$ & Penetrance, \% & $\begin{array}{l}\text { No. of genotyped genetically } \\
\text { informative } F 5^{L / L} \mathrm{Tfpi}^{+/-} \text {Mice }\end{array}$ \\
\hline MF5L1 & $M$ & 654 & 184 & 470 & 78.3 & 27 \\
\hline MF5L2 & $\mathrm{F}$ & 14 & 1 & 13 & 15.4 & 0 \\
\hline MF5L3 & $\mathrm{F}$ & 50 & 3 & 47 & 12.8 & 0 \\
\hline MF5L4 & $M$ & 3 & 1 & 2 & 100.0 & 0 \\
\hline MF5L5 & $M$ & 255 & 50 & 205 & 48.8 & 0 \\
\hline MF5L6 & M & 1,393 & 336 & 1,057 & 63.6 & 98 \\
\hline MF5L7 & $\mathrm{F}$ & 42 & 1 & 41 & 4.9 & 0 \\
\hline MF5L8 & $M$ & 543 & 132 & 411 & 64.2 & 0 \\
\hline MF5L9 & $M$ & 1,127 & 264 & 863 & 61.2 & 84 \\
\hline MF5L10 & $M$ & 111 & 15 & 96 & 31.3 & 0 \\
\hline MF5L11 & M & 459 & 121 & 338 & 71.6 & 0 \\
\hline MF5L12 & $M$ & 200 & 46 & 154 & 59.7 & 0 \\
\hline MF5L13 & $M$ & 115 & 13 & 102 & 25.5 & 0 \\
\hline MF5L14 & $M$ & 47 & 3 & 44 & 13.6 & 0 \\
\hline MF5L15 & $\mathrm{F}$ & 40 & 3 & 37 & 16.2 & 0 \\
\hline MF5L16 & $M$ & 442 & 119 & 323 & 73.7 & 14 \\
\hline
\end{tabular}

Penetrance was calculated as total $F 5^{\mathrm{LL}} \mathrm{Tfpi}^{{ }^{+/-}}$divided by half of the number of $F 5^{\mathrm{LL}} \mathrm{Tfpi}^{{ }^{\prime++}}$ littermates.

more than one suppressor mutation, the absence of a clear linkage signal for each of these lines likely reflects complex mouse strain modifier gene interactions, which are known to significantly impact mouse phenotypes $(10,27)$ and confound linkage analysis (28). Consistent with this hypothesis, we have previously documented poorer survival to weaning in mixed B6-129S1 $F 5^{L / L}$ mice compared with littermates (14). We extended these observations by the analysis of additional $F 5^{L /+}$ and $F 5^{L / L}$ littermates, with mice of the $F 5^{L / L}$ genotype demonstrating a $50 \%$ reduction in survival in the 129S1 versus B6 strain backgrounds (Table S1).
MF5L6 was maintained for 12 generations on both the mixed and B6 backgrounds and produced a total of $336 \mathrm{F5}^{\mathrm{L} / \mathrm{L}} \mathrm{Tfpi}{ }^{+/-}$mice (98 on the mixed B6-129S1 background and therefore useful for linkage analysis; see Table 1). Genome-wide SNP genotyping was performed on DNA from these 98 genetically informative $F 5^{L / L}$ $\mathrm{Tfpi}^{+/-}$mice; multipoint linkage analysis is shown in Fig. $2 A$. Since the genetic intervals around the F5 and Tfpi loci cannot be accurately assessed for linkage, these regions of chromosomes (Chr) 1 and 2 were excluded from linkage analysis (Materials and Methods and Fig. 2A). A single locus with a significant LOD score of
A

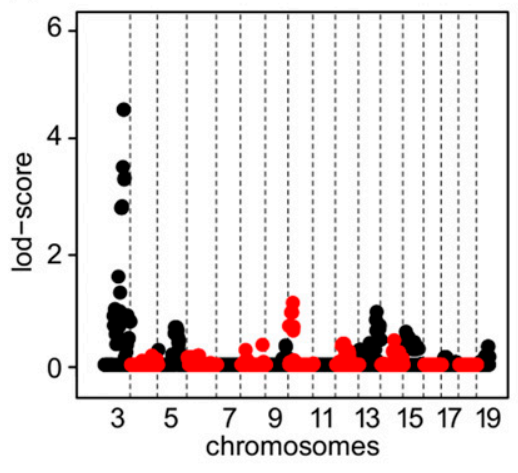

C
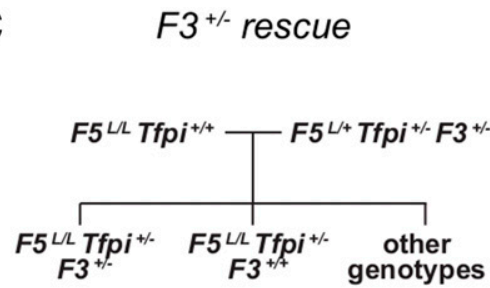

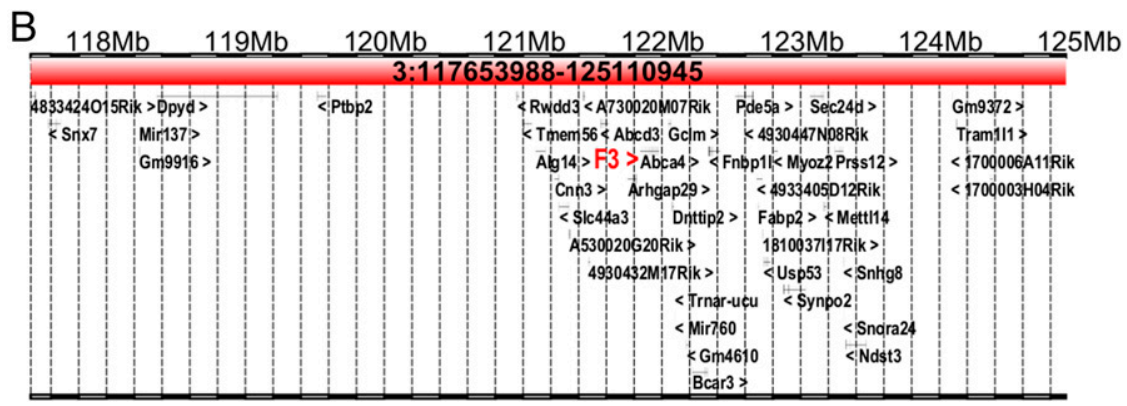

Fig. 2. The MF5L6 suppressor locus maps to Chr3. (A) Linkage analysis for the MF5L6 line. Alternating red and black are used to highlight the chromosomes. Chrs 1 and 2 were excluded from further analysis since they contain the $F 5$ and Tfpi genes, whose segregation was restricted by required genotypes at these loci. The Chr3 peak had the highest LOD score in the Chr3 subregion: 117.3-124.8 Mb [maximum LOD = 4.49, one LOD interval, significance threshold of LOD >3.3 (44)]. $(B)$ The Chr3 candidate interval (Chr3:117.3-124.8 Mb) contains 43 RefSeq-annotated genes, including $F 3$. (C) The mating scheme and observed distribution of offspring to test $F 3$ deficiency as a suppressor of $F 5^{L / L} \mathrm{Tfpi}^{+/-}$. $\mathrm{FS}^{+/-}$results in incompletely penetrant suppression of the $F 5^{L / L} \mathrm{Tfpi}^{+/-}$phenotype. 
4.49 was identified on Chr 3, with the 1 LOD interval (117.3$124.8 \mathrm{Mb}$ ) containing 43 National Center for Biotechnology Information reference sequence (RefSeq)-annotated genes (Fig. $2 B$ ).

The $F 3$ gene located within this interval (Chr3:121.7 Mb) (Fig. $2 B$ ) encodes tissue factor (TF), a procoagulant component of the hemostatic pathway that has Tfpi as its major regulator. Quantitative or qualitative deficiencies in $F 3$ are thus highly plausible candidates to suppress the $F 5^{L / L} T f p i^{+/-}$phenotype. To test $F 3$ as a candidate suppressor of the $F 5^{L / L} \mathrm{Tfpi}^{+/-}$phenotype, an independent $F 3$-null allele was introduced, and triple-heterozygous $\mathrm{F5}^{\mathrm{L} /+} \mathrm{Tfpi}^{+/-} \mathrm{F3}^{+/-}$mice were crossed to $F 5^{L / L}$ B6 mice (Fig. 2C). Of 273 progeny genotyped at weaning, $13 \mathrm{Fs}^{\mathrm{L} / L} \mathrm{Tfpi}^{+/-} \mathrm{F3}^{+/-}$mice and one $F 5^{L / L} \mathrm{Tfpi}^{+/-} \mathrm{FH}^{+/+}$mouse $\left(P=9.7 \times 10^{-5}\right)$ were observed. We also observed significantly fewer male than female $F 5^{L / L} \mathrm{Tfpi}^{+/-}$ $F 3^{+/-}$mice ( 2 vs. $11, P=0.03$ ). Thus, haploinsufficiency for $F 3^{+/-}$ suppresses the synthetic lethal $F 5^{L / L} \mathrm{Tfpi}^{+/-}$phenotype with incomplete penetrance $(33 \%)$ that also differs by sex $(10 \%$ for males and $67 \%$ for females). In contrast, the MF5L6 line exhibited an overall penetrance of $72.4 \%$, with similar male/female penetrance. Gender-specific differences in venous thrombosis rates have previously been reported, including contributions from oral contraceptives and hormone replacement therapy (29-31). This difference in penetrance could be due to 129S1 strain effects in the MF5L6 line or differences between a F3 regulatory mutation in MF5L6 compared with the $F 3$ loss-of-function allele used here.

Whole-exome sequencing data analysis of a $F 5^{\mathrm{L} / \mathrm{L}} \mathrm{Tfpi}^{+/-}$mouse from MF5L6 failed to identify an ENU variant in $F 3$ or in any other genes in the nonrecombinant interval or more broadly on the entire Chr3. This is a particularly gene-rich region (Fig. $2 B$ ), and errors in annotation could obscure the responsible variant. Of note, this interval also includes $S l c 44 a 3$, a paralog of $S l c 44 a 2$, the latter previously identified as a potential modifier of VTE risk in humans (6). Although additional ENU variants were identified on other chromosomes, none cosegregated with the survival phenotype in line MF5L6 (Table S2). Sanger sequencing analysis of the full set of F3 exons and introns, as well as $5 \mathrm{~kb}$ upstream of exon 1 , also failed to identify an ENU-induced mutation. In addition, analysis of $F 3$ mRNA levels in liver, lung, and brain tissues of adult mice failed to identify any differences in the level of expression from the ENUmutant compared with the WT allele (Fig. S1).

Taken together, these data suggest that an ENU-induced F3 regulatory mutation outside the sequenced segment may be responsible for thrombosuppression in MF5L6, although we cannot exclude a regulatory mutation in another gene. Nonetheless, our findings demonstrate that F3/Tfpi balance plays a key role in thrombosis in the mouse, particularly in the setting of $F 5^{L}$, and suggest that modest variations in either F3 or Tfpi could be important modifiers of VTE susceptibility in humans.

Whole-Exome Sequencing Identifies Candidate ENU Suppressor Variants for Eight MF5L Lines. Whole-exome next-generation sequencing (NGS) was performed on genomic DNA from an index $F 5^{L / L} \mathrm{Tfpi}^{+/-}$ mouse (from the G2-G5 generation) from each of eight $M F 5 L$ lines, including the four lines described above and four additional lines with large pedigrees (MF5L5, MF5L8, MF5L11, MF5L12). The mean coverage of sequenced exomes was more than $90 \times$, with $>97 \%$ of the captured region covered with at least six independent reads (Table S3). A total of 125 heterozygous variants were identified as candidate suppressor mutations, with 79 variants affecting protein sequence (Table S2). Of the total mutations, $54.4 \%$ were nonsynonymous single-nucleotide variants (SNVs), followed by UTRs $(17.6 \%)$ and synonymous $(14.4 \%)$ and stopgain SNVs (7.2\%), with the remainder being comprised of indels, splicing, and stop-loss mutations. The most common mutation events were $A / T \rightarrow G / C$ transitions $(35.3 \%$ ), while $C / G \rightarrow G / C$ transversions were the least represented $(2.5 \%)$. This spectrum of mutations is consistent with previously published ENU experiments (32). Variants exhibiting no recombination with the Tfpi locus on Chr2 (17 variants) were excluded from further analysis (Materials and Methods). Sanger sequencing confirmation was performed for 62 variants, including all nonsynonymous and stopgain mutations. These variants were then checked for parent of origin (either the G1 mutagenized progeny or its nonmutagenized

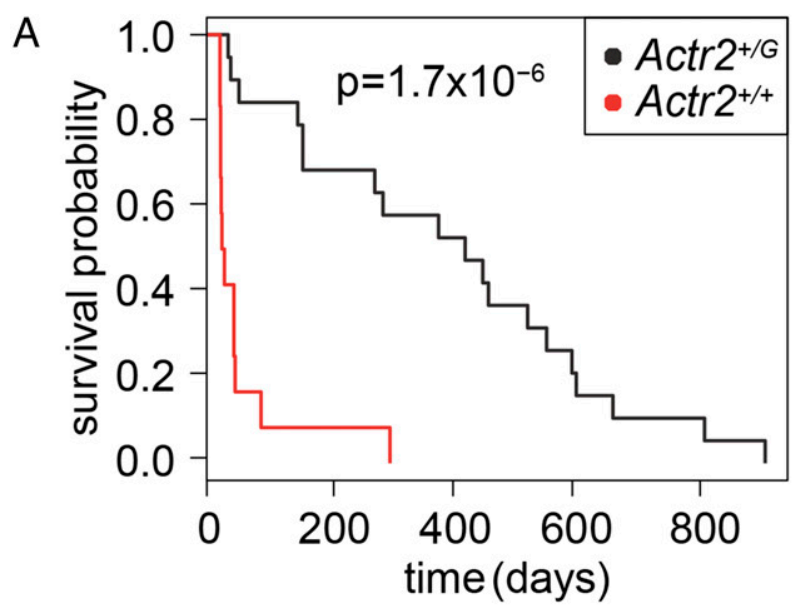

B

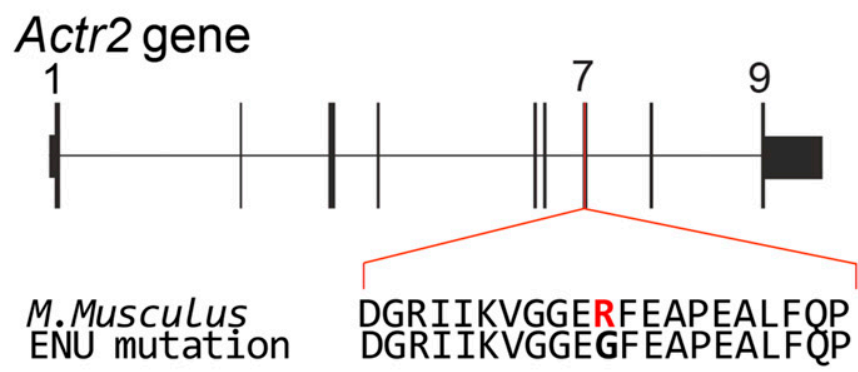
R. Norvegicus
O. Cuniculus
H.Sapiens
P.troglodytes
G. gorilla
S. scrofa
B. Taurus
A.melanoleuca
E.caballus
L. africano
M. Lucifugus
D. novemcintus
0 . anatinus
G.Gallus
A.Carolinensis
$X$. Tropicalis
G. Aculeatus
D. Rerio
D.Melanogaster
C.Elegans
S.Cerevisiae
A. Thaliana

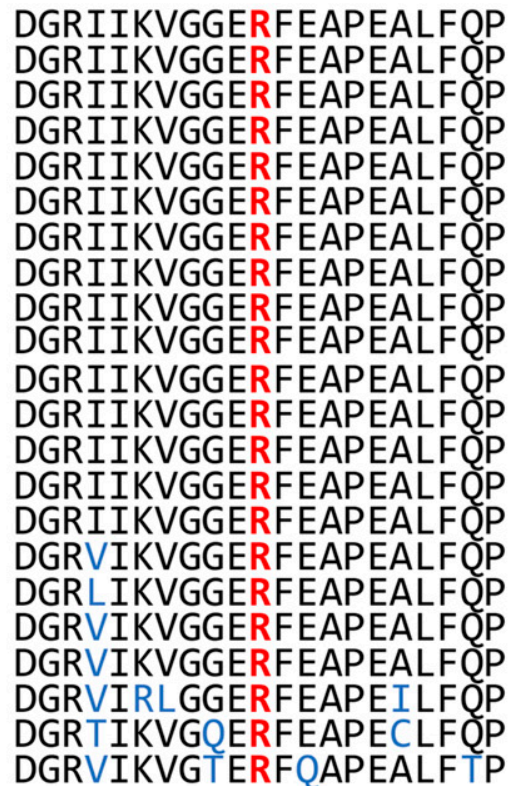

Fig. 3. The Actr2 R258G ENU-induced mutation is a potential thrombosis suppressor gene. (A) Kaplan-Meier survival plot for $\mathrm{F}^{L / L} \mathrm{Tfpi}^{+/-}$mice with and without the $A c t r 2^{G}$ mutation. F5 ${ }^{L / L} \mathrm{Tfpi}^{+/-}$Actr2 ${ }^{+/ G}$ mice exhibit significantly better survival than $\mathrm{FS}^{L / L} \mathrm{Tfpi}^{+/-} \mathrm{Actr}^{+/+}$littermates ( $n=19$ for Actr2 $^{+/ G}$, $n=12$ for Actr2 $^{+/+}, n=31$ total). (B) ARP2 amino acid R258 is highly conserved in animals, plants, and fungi. 
mate) as well as the original mutagenized G0 male. Forty-seven of these variants were identified in the G1 mouse but not in the G0 or nonmutagenized parent, consistent with ENU-induced mutations. The remaining 15 mutations were NGS sequencing errors $(11 / 15)$, de novo mutations $(2 / 15)$ or mutations transmitted from the nonmutagenized parent (2/15) (Table S2).

Each SNV was analyzed in additional $M F 5 L$ mice from the line in which it was identified. None of the thrombosuppressive exonic ENU-induced variants identified in lines MF5L1, 5, 6, 8, 9, 11, and 16 segregated with the lethal phenotype as tested by KaplanMeier analysis using a significance threshold of $P<0.05$ (33). Of the seven candidate ENU-induced SNVs identified from wholeexome sequence analysis for the MF5L12 line, one was an NGS sequencing error, and six were validated by Sanger sequencing as consistent with ENU-induced mutations in the G0 mice (Table S2). For each of these six SNVs, cosegregation with the survival phenotype was tested by Kaplan-Meier analysis of the first $31 F 5^{L / L}$ $\mathrm{Tfpi}^{+/-}$mice generated from the MF5L12 line. Only one variant, a nonsynonymous SNV in the actin-related protein 2 (Actr2) gene (c.772C > G, p.R258G, Actr $^{G}$ ), demonstrated a significant survival advantage when coinherited with the $F 5^{L / L} T f p i^{+/-}$genotype $\left(P=1.7 \times 10^{-6}\right)($ Fig. $3 A)$.

Actr2 as a Thrombosuppressor Gene. The gene Actr2 encodes the ARP2 protein, which is an essential component of the Arp2/3 complex (34). ARP2 along with ARP3 and five other independent protein subunits (ARPC1-5) form the evolutionarily conserved seven-subunit Arp2/3 complex (35). Arp2/3 is a major component of the actin cytoskeleton and is found in most eukaryotic cells, including platelets (36). Arp2/3 binds to the sides of actin filaments and initiates the growth of a new filament, leading to the creation of branched actin networks that are important for many cellular processes (37). Loss of Arp2/3 function can have severe consequences, as illustrated by the embryonic lethality of mice homozygous for an ARP3 hypomorph (38). In hemostasis, the Arp2/3 complex is necessary for actin-dependent platelet cytoskeletal remodeling events, which are essential for platelet activation and degranulation $(37,39$, 40). The $A c t r 2^{+/ G}$ mutation results in a p.R258G substitution in exon 7 of Actr 2 at a highly conserved amino acid position, with arginine present at this position for all 60 available vertebrate sequences (https://genome.ucsc.edu), as well as in plants and fungi (Fig. $3 B$ ). In addition, no variants at this position have been identified to date in over 120,000 human alleles (41).

Actr2 Hemizygosity Is Incompatible with Survival. We attempted to generate an independent $A c t r 2$ knockin $\left(A c t r 2^{G}\right)$ allele by CRISPR/ Cas9 genome editing (Materials and Methods and Figs. S2 and S3 and Table S4). Although highly efficient gene targeting was observed in blastocysts (Fig. S3), transfer of 275 injected embryos into foster mothers resulted in no surviving pups with a successfully targeted Actr2 allele. These data suggest that heterozygous loss of function for Actr2 may be incompatible with survival to term. Consistent with this hypothesis, human sequencing data from the Exome Aggregation Consortium (ExAC), which includes 60,706 individual exomes, reports a probability of loss-of-function intolerance for ACTR2 of 0.997 (41). ACTR2 mutations have not been previously associated with human disease (https://omim.org/entry/604221) (42), again as is consistent with early embryonic lethality. In addition, of 373,692 mouse ENU-induced mutations listed in the Mutagenetix website (https:// mutagenetix.utsouthwestern.edu/), only 16 are located in the Actr2 gene, with no predicted loss-of-function mutations (43). Taken together, these data strongly suggest that haploinsufficiency for Actr2 is not tolerated in humans or mice. The viability of $A c t r 2^{+/ G}$ mice

1. Silverstein MD, et al. (1998) Trends in the incidence of deep vein thrombosis and pulmonary embolism: A 25-year population-based study. Arch Intern Med 158:585-593.

2. Souto JC, et al. (2000) Genetic determinants of hemostasis phenotypes in Spanish families. Circulation 101:1546-1551. suggests that the $A c t r 2^{G}$ allele is either hypomorphic or a unique gainof-function mutation distinct from simple haploinsufficiency. Similarly, analysis of ExAC data suggests that four of the six other members of the Arp2/3 complex are intolerant of heterozygous loss of function in humans (41). Our findings suggest that subtle alterations in Actr2 function, and potentially in other components of the actin cytoskeleton, could alter hemostatic balance and play a previously unappreciated role in thrombosis susceptibility.

The identification of novel factors involved in the regulation of hemostasis is challenging; genes leading to marked shifts in hemostatic balance resulting in either severe bleeding or thrombosis are straightforward to identify clinically in humans, whereas subtle shifts are likely to escape detection given the multiple layers of buffering built into the complex hemostatic system (10). Homozygous deficiency (which would not be tested by our dominant suppressor strategy) for a number of hemostatic factors results in clinical bleeding, whereas heterozygous carriers remain asymptomatic. Although a single mutation in the X-chromosomal Factor VIII (or IX) gene produces severe bleeding in humans and rescues the lethal $F 5^{L / L}$ $\mathrm{Tfpi}^{+-}$mouse phenotype (Fig. $1 \mathrm{~A}$ ), an $F 8$ gene mutation would not be transmitted from the ENU-mutagenized male to male offspring and thus would be undetected. Indeed, the dominant sensitized suppressor screen reported here was undertaken to identify genes for which a modest $(\leq 50 \%)$ reduction in function would significantly shift the overall hemostatic balance. Such loci represent likely candidates for common human variation contributing to thrombosis and bleeding disorders. Gene variants with subtle yet significant antithrombotic effects represent attractive therapeutic targets because of a potentially wide therapeutic window with few unintended side effects. The finding of $98 \mathrm{F5}^{\mathrm{L} / \mathrm{L}} \mathrm{Tfpi}^{+/-}$mice carrying putative thrombosis suppressor mutations (at an estimated $1.5 \times$ genome coverage) suggests that subtle alterations at a number of loci are capable of suppressing the $F 5^{L / L} T f p i^{+/-}$lethal thrombotic phenotype. The complex strainspecific genetic modifiers that confounded the genetic linkage analysis are consistent with this model. Nonetheless, our findings illustrate the particular importance of the F3/Tfpi axis in thrombosis regulation (especially in the setting of $F 5^{L}$ ) as well as the identification of Actr2 and the Arp $2 / 3$ complex as another potentially sensitive regulatory pathway for maintaining hemostatic balance.

\section{Materials and Methods}

The University of Michigan Institutional Committee on the Use and Care of Animals approved all experiments using mice (protocol numbers PRO00007371, PRO00005191, and PRO00005913). Detailed descriptions of mouse strains and procedures for ENU mutagenesis, breeding, genetic mapping and genotyping, Sanger and whole-exome sequencing, estimation of $F 3$ allelic expression, generation of Actr2 CRISPR/Cas9-targeted mice and cells, the SURVEYOR nuclease assay, and statistical data analyses are provided in Supporting Information. Primers used in these studies are listed in Table S5.

ACKNOWLEDGMENTS. We thank the expertise of the Transgenic Animal Model Core staff of the University of Michigan's Biomedical Research Core Facilities for their assistance with this study. Research reported in this publication was supported by the National Cancer Institute of the NIH under Award P30CA046592 by the use of the following Cancer Center Shared Resources: Transgenic Animal Models. This research was supported by NIH Grants P01HL057346 (to D.G.) and R15-HL133907 and R01-HL135035 (to R.J.W.). R.J.W. was supported by the Oakland University Research Excellence Fund, an Aniara Diagnostica Coagulation Research Grant, an American Heart Association (AHA) Predoctoral Fellowship, and AHA Innovative Research and Scientist Development Grants. K.T. was an International Fulbright Science and Technology Fellow and the recipient of an AHA Predoctoral Fellowship. M.A.B. and A.J.J. were recipients of AHA Undergraduate Fellowships. D.G. is a member of the University of Michigan Cancer Center and is an Investigator of the Howard Hughes Medical Institute. The content is solely the responsibility of the authors and does not necessarily represent the official views of the $\mathrm{NIH}$.

3. Trégouët DA, et al. (2016) Is there still room for additional common susceptibility alleles for venous thromboembolism? J Thromb Haemost 14:1798-1802.

4. Dentali F, et al. (2012) Non-O blood type is the commonest genetic risk factor for VTE: Results from a meta-analysis of the literature. Semin Thromb Hemost 38:535-548. 
5. Rosendaal FR, Reitsma PH (2009) Genetics of venous thrombosis. J Thromb Haemost 7: 301-304.

6. Germain M, et al.; Cardiogenics Consortium (2015) Meta-analysis of 65,734 individuals identifies TSPAN15 and SLC44A2 as two susceptibility loci for venous thromboembolism. Am J Hum Genet 96:532-542.

7. Dahlbäck B (2008) Advances in understanding pathogenic mechanisms of thrombophilic disorders. Blood 112:19-27.

8. Lijfering WM, Rosendaal FR, Cannegieter SC (2010) Risk factors for venous thrombosis current understanding from an epidemiological point of view. BrJ Haematol 149:824-833.

9. Clark JS, Adler G, Salkic NN, Ciechanowicz A (2013) Allele frequency distribution of $1691 \mathrm{G}>\mathrm{A}$ F5 (which confers Factor V Leiden) across Europe, including Slavic populations. J Appl Genet 54:441-446.

10. Westrick RJ, Ginsburg D (2009) Modifier genes for disorders of thrombosis and hemostasis. J Thromb Haemost 7:132-135.

11. Evaluation of Genomic Applications in Practice and Prevention (EGAPP) Working Group (2011) Recommendations from the EGAPP Working Group: Routine testing for Factor V Leiden (R506Q) and prothrombin (20210G $>$ A) mutations in adults with a history of idiopathic venous thromboembolism and their adult family members. Genet Med 13:67-76.

12. De Stefano V, et al. (1999) The risk of recurrent deep venous thrombosis among heterozygous carriers of both factor $V$ Leiden and the G20210A prothrombin mutation. N Engl J Med 341:801-806.

13. van Boven $\mathrm{HH}$, et al. (1996) Factor $\mathrm{V}$ Leiden (FV R506Q) in families with inherited antithrombin deficiency. Thromb Haemost 75:417-421.

14. Cui J, et al. (2000) Spontaneous thrombosis in mice carrying the factor $V$ Leiden mutation. Blood 96:4222-4226.

15. Eitzman DT, et al. (2002) Lethal perinatal thrombosis in mice resulting from the in teraction of tissue factor pathway inhibitor deficiency and factor $V$ Leiden. Circulation 105:2139-2142.

16. Cordes SP (2005) N-ethyl-N-nitrosourea mutagenesis: Boarding the mouse mutant express. Microbiol Mol Biol Rev 69:426-439.

17. Moresco EM, Li X, Beutler B (2013) Going forward with genetics: Recent technological advances and forward genetics in mice. Am J Pathol 182:1462-1473.

18. Bull KR, et al. (2013) Unlocking the bottleneck in forward genetics using whole-genome sequencing and identity by descent to isolate causative mutations. PLoS Genet 9:e1003219.

19. Buchovecky CM, et al. (2013) A suppressor screen in Mecp2 mutant mice implicates cholesterol metabolism in Rett syndrome. Nat Genet 45:1013-1020.

20. Tchekneva EE, et al. (2007) A sensitized screen of N-ethyl-N-nitrosourea-mutagenized mice identifies dominant mutants predisposed to diabetic nephropathy. J Am Soc Nephrol 18:103-112.

21. Matera I, et al. (2008) A sensitized mutagenesis screen identifies $\mathrm{Gli3}$ as a modifier of Sox10 neurocristopathy. Hum Mol Genet 17:2118-2131.

22. Carpinelli MR, et al. (2004) Suppressor screen in Mpl-/- mice: c-Myb mutation cause supraphysiological production of platelets in the absence of thrombopoietin signaling. Proc Natl Acad Sci USA 101:6553-6558.

23. Bank I, et al. (2005) Elevated levels of FVIII:C within families are associated with an increased risk for venous and arterial thrombosis. J Thromb Haemost 3:79-84.

24. Nolan PM, et al. (2000) A systematic, genome-wide, phenotype-driven mutagenesis programme for gene function studies in the mouse. Nat Genet 25:440-443.

25. Justice MJ, et al. (2000) Effects of ENU dosage on mouse strains. Mamm Genome 11 484-488.

26. Davis AP, Justice MJ (1998) An Oak Ridge legacy: The specific locus test and its role in mouse mutagenesis. Genetics 148:7-12.

27. Lusis AJ (2012) Genetics of atherosclerosis. Trends Genet 28:267-275

28. Yoo YJ, Mendell NR (2008) The power and robustness of maximum LOD score statistics. Ann Hum Genet 72:566-574.

29. Kyrle PA, et al. (2004) The risk of recurrent venous thromboembolism in men and women. N Engl J Med 350:2558-2563.

30. Roach RE, et al. (2015) Sex difference in the risk of recurrent venous thrombosis: A detailed analysis in four European cohorts. J Thromb Haemost 13:1815-1822.

31. Vandenbroucke JP, et al. (2001) Oral contraceptives and the risk of venous thrombosis. N Engl J Med 344:1527-1535.
32. Justice MJ, Noveroske JK, Weber JS, Zheng B, Bradley A (1999) Mouse ENU mutagenesis. Hum Mol Genet 8:1955-1963.

33. Rich JT, et al. (2010) A practical guide to understanding Kaplan-Meier curves. Otolaryngol Head Neck Surg 143:331-336.

34. Rotty JD, Wu C, Bear JE (2013) New insights into the regulation and cellular functions of the ARP2/3 complex. Nat Rev Mol Cell Biol 14:7-12.

35. Rottner K, Hänisch J, Campellone KG (2010) WASH, WHAMM and JMY: Regulation of Arp2/3 complex and beyond. Trends Cell Biol 20:650-661.

36. Veltman DM, Insall RH (2010) WASP family proteins: Their evolution and its physiological implications. Mol Biol Cell 21:2880-2893.

37. Falet $\mathrm{H}$, et al. (2002) Importance of free actin filament barbed ends for Arp2/3 com plex function in platelets and fibroblasts. Proc Natl Acad Sci USA 99:16782-16787.

38. Vauti $F$, et al. (2007) Arp3 is required during preimplantation development of the mouse embryo. FEBS Lett 581:5691-5697.

39. Li Z, Kim ES, Bearer EL (2002) Arp2/3 complex is required for actin polymerization during platelet shape change. Blood 99:4466-4474.

40. Koseoglu S, et al. (2015) VAMP-7 links granule exocytosis to actin reorganization during platelet activation. Blood 126:651-660.

41. Lek M, et al.; Exome Aggregation Consortium (2016) Analysis of protein-coding genetic variation in 60,706 humans. Nature 536:285-291.

42. McKusick VA (2007) Mendelian inheritance in man and its online version, OMIM. Am Hum Genet 80:588-604.

43. Beutler B (2017) MUTAGENETIX. Available at www.mutagenetix.net/home.cfm. Accessed April 6, 2017

44. Lander E, Kruglyak L (1995) Genetic dissection of complex traits: Guidelines for interpreting and reporting linkage results. Nat Genet 11:241-247.

45. Toomey JR, Kratzer KE, Lasky NM, Stanton JJ, Broze GJ, Jr (1996) Targeted disruption of the murine tissue factor gene results in embryonic lethality. Blood 88:1583-1587.

46. Huang ZF, Higuchi D, Lasky N, Broze GJ, Jr (1997) Tissue factor pathway inhibitor gene disruption produces intrauterine lethality in mice. Blood 90:944-951.

47. Bi L, et al. (1995) Targeted disruption of the mouse factor VIII gene produces a mode of haemophilia A. Nat Genet 10:119-121.

48. Lange K, et al. (2013) Mendel: The Swiss army knife of genetic analysis programs. Bioinformatics 29:1568-1570.

49. Mohlke KL, et al. (1999) Mvwf, a dominant modifier of murine von Willebrand factor, results from altered lineage-specific expression of a glycosyltransferase. Cell 96:111-120.

50. Tomberg K, et al. (2016) Spontaneous 8 bp deletion in Nbeal2 recapitulates the gray platelet syndrome in mice. PLoS One 11:e0150852.

51. Li H, Durbin R (2009) Fast and accurate short read alignment with Burrows-Wheeler transform. Bioinformatics 25:1754-1760.

52. García-Alcalde F, et al. (2012) Qualimap: Evaluating next-generation sequencing alignment data. Bioinformatics 28:2678-2679.

53. DePristo MA, et al. (2011) A framework for variation discovery and genotyping using next-generation DNA sequencing data. Nat Genet 43:491-498.

54. Wang K, Li M, Hakonarson H (2010) ANNOVAR: Functional annotation of genetic variants from high-throughput sequencing data. Nucleic Acids Res 38:e164.

55. Ran FA, et al. (2013) Genome engineering using the CRISPR-Cas9 system. Nat Protoc 8 : 2281-2308.

56. Brinster RL, Chen HY, Trumbauer ME, Yagle MK, Palmiter RD (1985) Factors affecting the efficiency of introducing foreign DNA into mice by microinjecting eggs. Proc Nat Acad Sci USA 82:4438-4442.

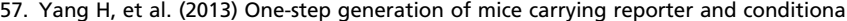
alleles by CRISPR/Cas-mediated genome engineering. Cell 154:1370-1379.

58. Sakurai T, Watanabe S, Kamiyoshi A, Sato M, Shindo T (2014) A single blastocyst assay optimized for detecting CRISPR/Cas9 system-induced indel mutations in mice. $B M C$ Biotechnol 14:69.

59. Therneau TM, Grambsch PM (2000) Modeling Survival Data: Extending the Cox Model (Springer, New York), 1st Ed, pp xiv, 350.

60. Brinkman EK, Chen T, Amendola M, van Steensel B (2014) Easy quantitative assessment of genome editing by sequence trace decomposition. Nucleic Acids Res 42:e168.

61. Singh P, Schimenti JC, Bolcun-Filas E (2015) A mouse geneticist's practical guide to CRISPR applications. Genetics 199:1-15. 